\title{
Steady-State and Time-Dependent Fluorescence Quenching Studies of the Binding of Anionic Micelles to Polycation ${ }^{\dagger}$
}

\author{
Akihito Hashidzume,* Katsunori Yoshida, \\ Department of Macromolecular Science, Graduate School of Science, Osaka University, \\ Toyonaka, Osaka 560-0043, Japan \\ Paul L. Dubin* \\ Department of Chemistry, Indiana University-Purdue University, Indianapolis, Indiana 46205-2820
}

Received: May 30, 2001

Soluble complex formation between poly( $N$-methyl-4-vinylpyridinium chloride) (QPVP) and mixed micelles of dodecyl octa(ethylene glycol) monoether $\left(\mathrm{C}_{12} \mathrm{E}_{8}\right)$ and sodium dodecyl sulfate (SDS), driven by electrostatic interactions in water, was investigated by turbidimetric, quasielastic light scattering, and fluorescence techniques. The polymer-micelle interaction was monitored through quenching of fluorescence of pyrene probes solubilized in $\mathrm{C}_{12} \mathrm{E}_{8} / \mathrm{SDS}$ mixed micelles, the quenching occurring upon binding of the micelles to QPVP. Unlike the case in which fluorescence-labeled polyelectrolytes are employed in conjunction with quencher-carrying micelles to monitor polymer-micelle interactions, QPVP plays a dual role as a polycation to interact with anionic micelles and a quencher for fluorescence of a probe solubilized in the micelle. Thus, hydrophobic contribution from fluorescence labels, an additional complexity in the former case, can be eliminated by employing QPVP, making it possible to monitor polymer-micelle interactions without hydrophobic effects. The presence of a well-defined critical mole fraction of SDS $\left(Y_{\mathrm{c}}\right)$ at which a soluble polymer-micelle complex formation begins to occur was confirmed. The $Y_{\mathrm{c}}$ values determined by turbidimetric and fluorescence titrations were found to be in good agreement. Dynamic interactions of QPVP with pyrene-carrying $\mathrm{C}_{12} \mathrm{E}_{8} / \mathrm{SDS}$ mixed micelles were monitored by steady-state and time-dependent fluorescence quenching techniques. The charge on the micelle was varied systematically by varying the mole fraction of $\operatorname{SDS}(Y)$ in the mixed micelle. Steady-state and time-dependent fluorescence-quenching data were analyzed using, as a first approximation, a kinetic model proposed previously. The lifetime of the micelle bound to the polymer (i.e., the residence time) was estimated as a function of the micelle surface charge density and ionic strength. Results revealed that the residence time is a strong function of both $Y$ and the ionic strength.

\section{Introduction}

Interactions between polyelectrolytes and oppositely charged micelles normally lead to macroscopic phase separation in water. ${ }^{1}$ However, polyelectrolyte- micelle interactions may lead to the formation of stable equilibrium complexes if the electrostatic attractions are attenuated by proper adjustment of the polyion linear charge density $(\xi)$, the micelle surface charge density $(\sigma)$, or the ionic strength $(\mu)$. Under properly adjusted conditions, soluble complexes with dimensions between 1 and 10 times those of the polyelectrolyte may be formed. ${ }^{2-6}$

The interaction of micelles with oppositely charged polyelectrolytes strongly resembles the interaction of polyelectrolytes with other particles of similar size and charge, such as proteins $^{7-11}$ and dendrimers. ${ }^{12-14}$ In all these cases, complex formation occurs when $\sigma$ reaches an adequate level, and the magnitude of this value varies nearly directly with $\mu^{a}(a \approx 1 / 2)$ and inversely with $\xi$. The appearance of the complexed state is sufficiently abrupt to enable the identification of a critical surface charge density $\left(\sigma_{\mathrm{c}}\right)$, so that the foregoing observations may be expressed as

\footnotetext{
† Part of the special issue "Noboru Mataga Festschrift".

$\doteqdot$ Current address: Shiseido Basic Research Center, 2-2-1 Hayabuchi, Tsuzuki, Yokohama 224-8558, Japan
}

$$
\sigma_{\mathrm{c}} \propto \xi^{-1} \kappa^{b}
$$

where $\kappa$ is the Debye-Hückel parameter. The value of $b$ may depend somewhat on micelle curvature and polyelectrolyte charge density ${ }^{15}$ but for strong polyelectrolytes in combination with small micelles is in the range of $1.0<b<1.4 .{ }^{15,16}$ The observation of such phase-transition-like behavior is consistent with theoretical predictions ${ }^{17-21}$ and simulations. ${ }^{22}$ On the other hand, the measured quantities, the departures of which from preassociation levels are thought to signal the onset of complex formation (vide infra), display changes that are never truly discontinuous but instead vary in abruptness depending on the polyelectrolyte-micelle system.

These soluble polymer-micelle complexes have been investigated by various characterization methods, including turbidimetry, ${ }^{23-26}$ dynamic and static light scattering, ${ }^{2-6}$ viscometry, ${ }^{6,27}$ electrophoretic light scattering, ${ }^{6}$ microcalorimetry, ${ }^{28}$ dyesolubilization, ${ }^{29,30}$ equilibrium dialysis, ${ }^{2,31,32}$ and fluorescence. ${ }^{33-36}$ Among these methods, fluorescence techniques are expected to provide a powerful tool to gain insight into the dynamics of polyelectrolyte-micelle interactions. Recently, we used a pyrene-labeled polyanion in conjunction with quencher-carrying mixed micelles to characterize the microscopic polyelectrolytemicelle phase transition. ${ }^{33-36}$ The intensity of the polyelectro- 


\section{CHART 1}

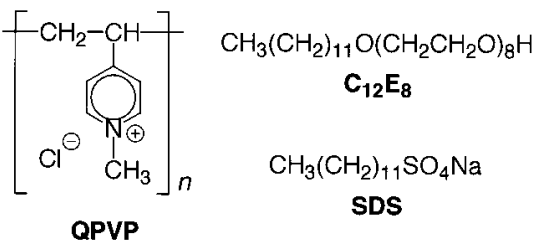

lyte-micelle interaction in such systems may be modulated by controlling the molar ratio of cationic and nonionic surfactants in the micelle (i.e., $\sigma$ ) and also by controlling the ionic strength (i.e., $\kappa)$. The enhancement of quenching arising from dynamic interactions between a pyrene-labeled polyanion and quenchercarrying mixed micelles upon increase in $\sigma$ or decrease in $\kappa$ was investigated by steady-state and time-dependent fluorescence spectroscopy..$^{33-36}$ Of particular importance is kinetic information available from the latter technique. While the rate of change of, for example, light scattering or viscosity in the vicinity of "critical conditions" may be complicated by alterations in complex structure after binding per se, the measurement of micelle-polyion residence times at $\sigma_{\mathrm{c}}$ might provide more direct insight into the meaning of the observed transition-like behavior.

The behavior of fluorescence-labeled polyelectrolytes may be perturbed by the physicochemical properties of the label. Turbidimetric, light scattering, and fluorescence studies using a copolymer of sodium 2-(acrylamido)-2-methylpropanesulfonate (AMPS) (99 mol \%) and $N$-(1-pyrenylmethyl)methacrylate (PyMAm) (1 mol \%), PyPAMPS, as a pyrenelabeled polyanion revealed that the interaction of PyPAMPS with mixed micelles of dodecyl hexa(ethylene glycol) monoether $\left(\mathrm{C}_{12} \mathrm{E}_{6}\right)$ and cetyltrimethylammonium chloride (CTAC) occurred preferentially with pyrene sites, although the interaction was predominantly driven by electrostatic attractions. ${ }^{33-35}$ These results indicate a conjoint effect of hydrophobic and electrostatic interactions on the polyion-micelle interaction. ${ }^{33-35}$ The preferential binding of $\mathrm{C}_{12} \mathrm{E}_{6} / \mathrm{CTAC}$ mixed micelles to hydrophobic sites incorporated into a polyanion was confirmed in a systematic study using terpolymers of AMPS, PyMAm (1 mol \%), and $N$-dodecylmethacrylamide $(0-7.5 \mathrm{~mol} \%)$ in conjunction with $\mathrm{C}_{12} \mathrm{E}_{6} / \mathrm{CTAC}$ mixed micelles in which cetylpyridinium chloride (CPC) is solubilized. ${ }^{36}$ All of these results indicate that when fluorescence-labeled polyelectrolytes are employed to investigate the dynamics of the complex formation, the hydrophobic contributions from fluorescence-label sites are inevitable, although fluorescence quenching techniques are a powerful tool for studies of polyelectrolyte-micelle interactions.

To avoid hydrophobic contribution arising from fluorescence labels, we used poly( $N$-methyl-4-vinylpyridinium chloride) (QPVP) (Chart 1) as a polyelectrolyte to interact with mixed micelles of nonionic and anionic surfactants. Pyridinium cations are known to be an efficient quencher for singlet-excited pyrene by means of an electron-transfer mechanism. ${ }^{37}$ Thus, QPVP is expected to play a dual role as a polycation and a quencher when the polymer interacts with pyrene-carrying micelles.

In this paper, we report on static and dynamic aspects of soluble complex formation between QPVP and mixed micelles of dodecyl octa(oxyethylene) glycol monoether $\left(\mathrm{C}_{12} \mathrm{E}_{8}\right)$ and sodium dodecyl sulfate (SDS) (Chart 1) studied by means of turbidimetric titration, quasielastic light scattering, and fluorescence measurements in which molecular pyrene is solubilized in $\mathrm{C}_{12} \mathrm{E}_{8} / \mathrm{SDS}$ mixed micelles. A previously proposed kinetic model $^{35,36}$ was used to interpret steady-state and time-dependent fluorescence quenching data, which made it possible to examine the residence time of the micelle in the polymer-micelle complex as a function of the micelle charge density and the ionic strength. Such measurements may be directly comparable to the results of Monte Carlo simulations. ${ }^{22}$

\section{Experimental Section}

Materials. Poly( $N$-methyl-4-vinylpyridinium chloride) (QPVP) was prepared from poly(4-vinylpyridine) $\left(M_{\mathrm{w}}=7.7 \times 10^{4}\right)$ obtained from Reilly Industries (Indianapolis). ${ }^{38}$ Dodecyl octa(ethylene glycol) monoether $\left(\mathrm{C}_{12} \mathrm{E}_{8}\right)$ (Nikko Chemical), purity confirmed by high performance liquid chromatography, was used as received. Sodium dodecyl sulfate (SDS) (Nakalai tesque) was recrystallized twice from methanol. Sodium chloride $(\mathrm{NaCl})$ (Wako) was used without further purification. Milli-Q water was used for all experiments.

Turbidimetric Titration. Turbidimetric titrations were carried out at $450 \mathrm{~nm}$ with a Brinkmann PC800 probe colorimeter equipped with a $2 \mathrm{~cm}$ path length fiber optics probe. "Type 1" turbidimetric titrations ${ }^{23-26}$ were performed at $25 \pm 1{ }^{\circ} \mathrm{C}$ by adding an aqueous solution of $40 \mathrm{mM}$ SDS at a constant ionic strength $(\mu)$ to a solution of $0.62 \mathrm{~g} / \mathrm{L}$ ( $4 \mathrm{mM}$ monomer units) QPVP and $20 \mathrm{mM} \mathrm{C}_{12} \mathrm{E}_{8}$ of the same $\mu$. The values of $\mu$ were adjusted with $\mathrm{NaCl}$. All transmittance values were corrected by subtracting the turbidity of a polymer-free blank. The blankcorrected turbidity $(100-\% T)$ was plotted as a function of $Y$, the mole fraction of the anionic surfactant in the mixed micelle, defined as $Y=[\mathrm{SDS}] /\left(\left[\mathrm{C}_{12} \mathrm{E}_{8}\right]+[\mathrm{SDS}]\right)$. "Type 2" turbidimetric titrations ${ }^{33,39,40}$ were carried out $25 \pm 1{ }^{\circ} \mathrm{C}$ by adding an aqueous solution of $100 \mathrm{mM} \mathrm{C}_{12} \mathrm{E}_{8} / \mathrm{SDS}$ mixed micelle at a constant $Y$ and a constant $\mu$ to a solution of $0.16 \mathrm{~g} / \mathrm{L}(1 \mathrm{mM}$ monomer units) QPVP of the same $\mu$. The values of $\mu$ were adjusted with $\mathrm{NaCl}$. The turbidity was plotted as a function of the concentration of total added surfactant $\left(C_{\mathrm{s}}\right)$.

Quasielastic Light Scattering (QELS). QELS measurements were carried out with a DynaPro 801 (Protein Solution Inc.), which employs a 30-mW solid-state 780-nm laser and an avalanche photodiode detector. Sample solutions were introduced into a $7-\mu \mathrm{L}$ cell through a $0.1-\mu \mathrm{m}$ Anotec filter. The $90^{\circ}$ scattering data were analyzed employing cumulants to determine the apparent hydrodynamic radii $\left(R_{\mathrm{h}}\right)$. Sample solutions were prepared by adding a 40-mM SDS solution at a constant $\mu$ to a solution of $20 \mathrm{mM} \mathrm{C}_{12} \mathrm{E}_{8}$ and $0.62 \mathrm{~g} / \mathrm{L}$ ( $4 \mathrm{mM}$ monomer units) QPVP of the same $\mu$.

Fluorescence. Steady-state fluorescence spectra were recorded on a Hitachi F-4500 fluorescence spectrophotometer with excitation at $338 \mathrm{~nm}$.

A pyrene-carrying $\mathrm{C}_{12} \mathrm{E}_{8}$ micelle stock solution was prepared as follows: About $20 \mu \mathrm{L}$ of an acetone solution of $13 \mathrm{mM}$ pyrene was placed in a $20 \mathrm{~mL}$ volumetric flask. After evaporation of the solvent, pyrene was dissolved in $\mathrm{C}_{12} \mathrm{E}_{8}(647.9 \mathrm{mg}$, $1.20 \mathrm{mmol}$ ), and then a predetermined concentration of aqueous $\mathrm{NaCl}$ was added to the mixture. The solution was stirred overnight. The concentration of pyrene was determined by absorption spectroscopy using $\epsilon=2.9 \times 10^{4} \mathrm{M}^{-1} \mathrm{~cm}^{-1}$ determined for pyrene in 4:1 (v/v) ethanol/water.

For "type 1" fluorescence titration, ${ }^{33-36}$ a solution of $40 \mathrm{mM}$ $\mathrm{SDS}$ in a predetermined concentration of $\mathrm{NaCl}$ was added to a solution containing $0.62 \mathrm{~g} / \mathrm{L}$ ( $4 \mathrm{mM}$ monomer units) QPVP and $20 \mathrm{mM}$ pyrene-tagged $\mathrm{C}_{12} \mathrm{E}_{8}$ at a constant ionic strength.

Fluorescence decays were measured by a time-correlated single-photon counting technique using a Horiba NAES 550 system. Decay curves were analyzed by a conventional decon- 


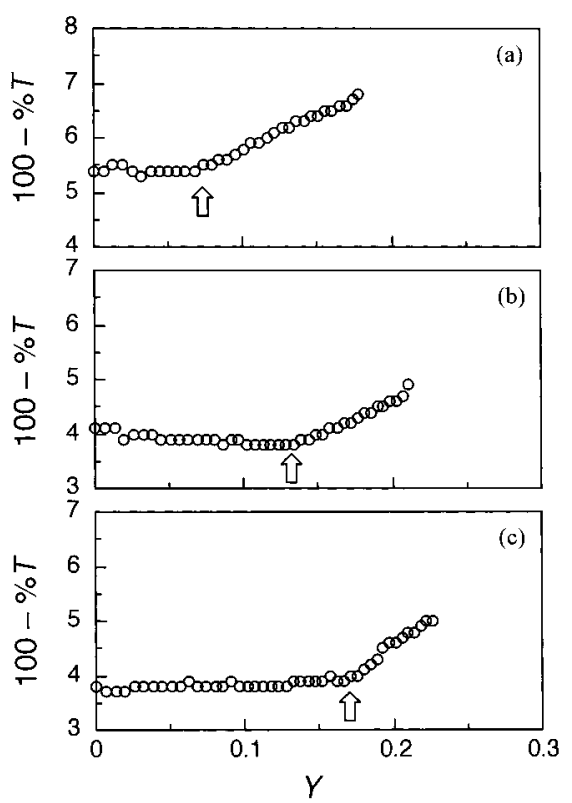

Figure 1. Type 1 turbidimetric titrations for $0.62 \mathrm{~g} / \mathrm{L}$ ( $4 \mathrm{mM}$ monomer units) QPVP and $20 \mathrm{mM} \mathrm{C}_{12} \mathrm{E}_{8}$ in 0.1 (a), 0.2 (b), and $0.3 \mathrm{M} \mathrm{NaCl}$ (c), using a $40 \mathrm{mM}$ SDS solution of the same $\mu$. The SDS solution was titrated to the mixed solution of the polymer and the micelle at a constant $\mu . Y$ is the mole fraction of SDS in the mixed micelle. Arrows denote $Y_{\mathrm{c}}$ values.

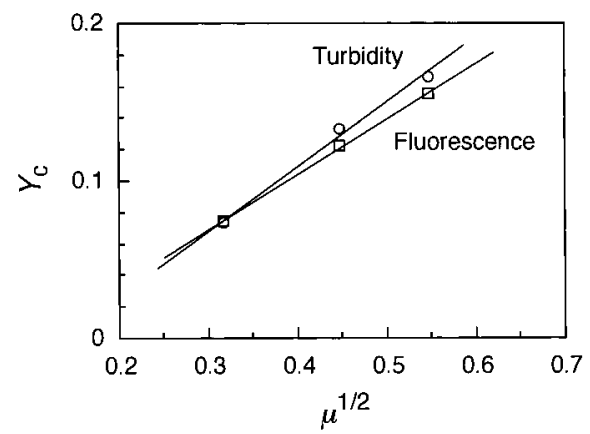

Figure 2. Dependence of $Y_{\mathrm{c}}$, determined by turbidimetric and fluorescence titrations, on the square root of $\mu$.

volution technique. Sample solutions were the same as those used for the steady-state fluorescence measurements described above.

\section{Results and Discussion}

Type 1 Turbidimetric Titrations. Solutions containing 0.62 $\mathrm{g} / \mathrm{L}$ (4 mM monomer units) QPVP and $20 \mathrm{mM} \mathrm{C}_{12} \mathrm{E}_{8}$ were titrated with a $40-\mathrm{mM}$ SDS aqueous solution at varying $\mu$. Figure 1 shows the turbidity (reported as $100-\% T$ ) as a function of $Y$ (the mole fraction of SDS in $\mathrm{C}_{12} \mathrm{E}_{8} / \mathrm{SDS}$ mixed micelles). At $\mu=0.1$, the turbidity is nearly constant in the region of $0 \leq Y$ $\leq 0.07$ but it commences to increase at $Y=0.07$, indicative of complex formation between QPVP and $\mathrm{C}_{12} \mathrm{E}_{8} / \mathrm{SDS}$ mixed micelles. This $Y$ value is designated as $Y_{\mathrm{c}}$. As $\mu$ is increased from 0.1 to 0.2 and $0.3, Y_{\mathrm{c}}$ increases to 0.13 and 0.17 , respectively. These observations are qualitatively consistent with those found for a number of polyelectrolyte-mixed micelle systems reported so far. ${ }^{23-25,33,41,42}$ As shown in Figure 2, the dependence of $Y_{\mathrm{c}}$ on $\mu$ is roughly consistent with previous results for other polyelectrolyte-micelle systems. ${ }^{24,33,42}$ The number of measurements at different $\mu$ is not large enough to specify the exact dependence of $Y_{\mathrm{c}}$ on $\mu$, but the ionic strength effect

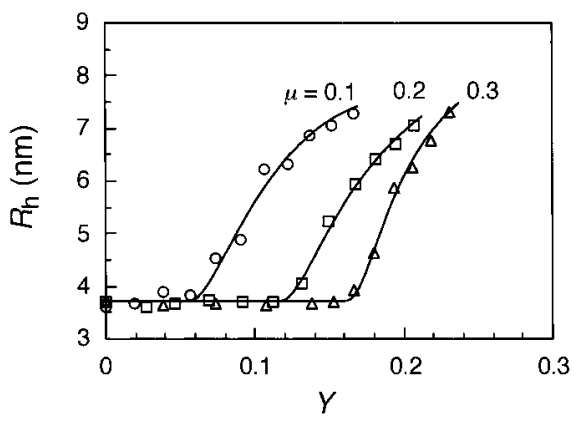

Figure 3. $R_{\mathrm{h}}$ as a function of $Y$ at varying $\mu . Y$ values were adjusted by adding a $40 \mathrm{mM}$ SDS solution to a solution containing $20 \mathrm{mM} \mathrm{C}_{12} \mathrm{E}_{8}$ and $0.62 \mathrm{~g} / \mathrm{L}$ QPVP.

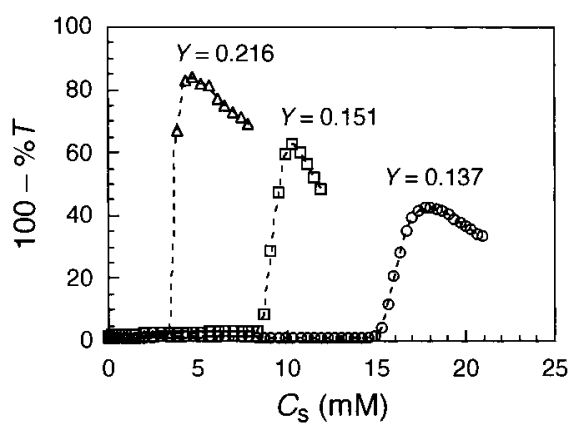

Figure 4. Type 2 turbidimetric titrations for $0.16 \mathrm{~g} / \mathrm{L}(1 \mathrm{mM}$ monomer units) QPVP in $0.1 \mathrm{M} \mathrm{NaCl}$, using a $100 \mathrm{mM} \mathrm{C} \mathrm{C}_{12} \mathrm{E}_{8} / \mathrm{SDS}$ solution of the same $\mu$. The solution of $\mathrm{C}_{12} \mathrm{E}_{8} / \mathrm{SDS}$ mixed micelles at a constant $Y$ was titrated to the QPVP at $\mu=0.1 . C_{\mathrm{s}}$ is the total surfactant concentration.

does suggest that the QPVP $-\mathrm{C}_{12} \mathrm{E}_{8} / \mathrm{SDS}$ interaction is driven by electrostatic attractions.

QELS of the QPVP- $\mathbf{C}_{12} \mathbf{E}_{8} /$ SDS System. QELS measurements were carried out to estimate the hydrodynamic size of QPVP $-\mathrm{C}_{12} \mathrm{E}_{8} / \mathrm{SDS}$ complexes. $Y$ values were adjusted by adding a $40 \mathrm{mM}$ SDS solution to a solution containing $20 \mathrm{mM}$ $\mathrm{C}_{12} \mathrm{E}_{8}$ and $0.62 \mathrm{~g} / \mathrm{L}$ QPVP. Figure 3 shows apparent values of $R_{\mathrm{h}}$ determined by cumulants as a function of $Y$ at varying ionic strengths. At all ionic strengths studied, $R_{\mathrm{h}}$ values are nearly constant in the region of $Y<Y_{\mathrm{c}}$.

$Y_{\mathrm{c}}$ values are estimated to be $0.06,0.12$, and 0.16 at $\mu=0.1$, 0.2 , and 0.3 , respectively, i.e., consistently about $5-10 \%$ smaller than the turbidimetric estimates, possibly indicating the higher sensitivity of the QELS results. The increase in $R_{\mathrm{h}}$ for $Y \geq Y_{\mathrm{c}}$ can arise either from a continuous increase in the size of complexes or from a shift in the population of scatterers, as the concentration of free micelles falls concomitant with an increase in the concentration of larger (but possibly uniform) complexes.

Type 2 Turbidimetric Titrations. Type 2 titrations, corresponding to the addition of mixed micelles at constant $Y$ to polymer solution at constant $\mu$, also provide information about the stoichiometry of complex formation. Type 2 turbidimetric titrations were conducted at $0.16 \mathrm{~g} / \mathrm{L}$ QPVP $+\mathrm{C}_{12} \mathrm{E}_{8} / \mathrm{SDS}$ mixed micelles in $0.1,0.2$, and $0.3 \mathrm{M} \mathrm{NaCl}$ at constant $Y$. Figure 4 shows the results of the type 2 turbidimetric titrations at $\mu=$ 0.1 , in which the turbidity is plotted as a function of $C_{\mathrm{s}}$. Even at a $Y$ sufficiently higher than $Y_{\mathrm{c}}(0.07)$, turbidity shows only a slight increase with $C_{\mathrm{s}}$ in the lower $C_{\mathrm{s}}$ region. When $C_{\mathrm{s}}$ is further increased beyond a certain value, turbidity increases remarkably, indicative of phase separation. As $Y$ is increased, the critical $C_{\mathrm{s}}$ decreases and the increase in turbidity at about the critical $C_{\mathrm{s}}$ becomes more abrupt. At $\mu=0.2$ and 0.3 , the tendencies are the same as that at $\mu=0.1$. Because the soluble complexes are 


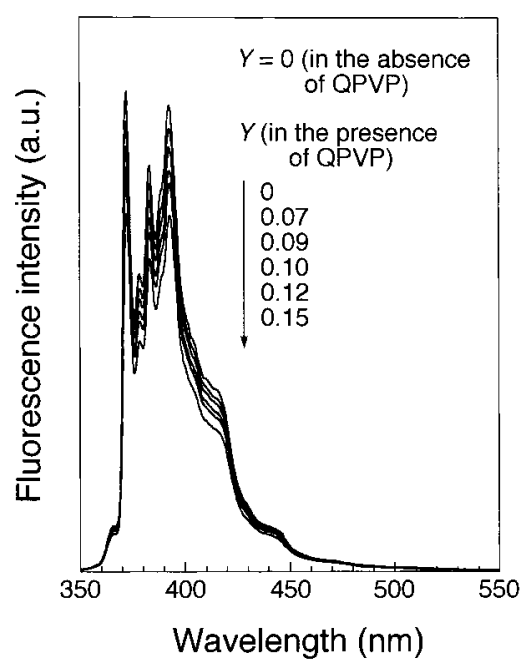

Figure 5. Fluorescence spectra of pyrene-carrying $\mathrm{C}_{12} \mathrm{E}_{8} / \mathrm{SDS}$ mixed micelles of varying $Y$ in the absence and presence of QPVP in $0.1 \mathrm{M}$ $\mathrm{NaCl}$. $Y$ values were adjusted by addition of $40 \mathrm{mM}$ SDS in $0.1 \mathrm{M}$ $\mathrm{NaCl}$ to a mixture of $0.62 \mathrm{~g} / \mathrm{L} \mathrm{QPVP}, 20 \mathrm{mM} \mathrm{C}_{12} \mathrm{E}_{8}$, and $4.6 \mu \mathrm{M}$ pyrene.

intrapolymer at low $C_{\mathrm{s}}$, the turbidity is small, indicating that soluble complexes are in association equilibrium even at a $Y$ much higher than $Y_{\mathrm{c}}$. Above the critical $C_{\mathrm{s}}$, highly turbid interpolymer complexes are formed presumably because of charge neutralization of the complexes, resulting in phase separation.

Type 1 Fluorescence Titrations. To monitor the polymermicelle interaction by fluorescence quenching, pyrene was dissolved in $\mathrm{C}_{12} \mathrm{E}_{8}$ /SDS micelles and fluorescence was monitored in the presence of QPVP as a function of $Y$. Figure 5 shows fluorescence spectra of pyrene solubilized in $\mathrm{C}_{12} \mathrm{E}_{8} / \mathrm{SDS}$ mixed micelles of varying $Y$ in the absence and the presence of QPVP in $0.1 \mathrm{M} \mathrm{NaCl}$. Values of $Y$ were adjusted by adding a $40 \mathrm{mM}$ SDS solution to a mixture of $0.62 \mathrm{~g} / \mathrm{L}$ QPVP, $20 \mathrm{mM}$ $\mathrm{C}_{12} \mathrm{E}_{8}$, and $4.6 \mu \mathrm{M}$ pyrene. Complete dissolution of pyrene in the micelle was confirmed by the fact that fluorescence decay is single-exponential with a lifetime of ca. $380 \mathrm{~ns}$, as will be discussed in detail in the following subsection. Figure 5 shows a significant decrease in the fluorescence intensity with increasing $Y$, indicating that the fluorescence quenching occurs upon complex formation. Figure 6 shows the $Y$-dependence of the normalized fluorescence intensity, $I / I_{0}$, where $I$ denotes the fluorescence intensity of the pyrene-carrying $\mathrm{C}_{12} \mathrm{E}_{8} / \mathrm{SDS}$ micelles at varying $Y$ in the presence of QPVP and $I_{0}$ denotes the fluorescence intensity in the absence of QPVP at $Y=0$. It was confirmed that in the absence of QPVP, the fluorescence intensity was independent of $Y$, i.e., the fluorescence intensity remains unchanged after adding SDS to pyrene-carrying $\mathrm{C}_{12} \mathrm{E}_{8}$ micelles (data not shown). At $\mu=0.1$, fluorescence intensities at $Y \leq 0.07$ are virtually the same as $I_{0}$. Fluorescence quenching is only observed at $Y>0.07$, and the extent of the quenching increases as $Y$ is increased. These observations provide evidence for the complex formation of QPVP with the pyrene-carrying micelle at $Y \geq Y_{\mathrm{c}}$ and an increase in the fraction of bound micelles with increasing micellar charge density. From the results of fluorescence quenching, $Y_{\mathrm{c}}$ values were determined to be $0.07,0.12$, and 0.16 at $\mu=0.1,0.2$, and 0.3 , respectively. As shown in Figure 2, these $Y_{\mathrm{c}}$ values are in good agreement with those determined by turbidimetric titration.

After $Y_{\mathrm{c}}$ is reached, quenching increases gradually and almost linearly with further increase in $Y$. The same behavior is reflected in Figure 1, in which the turbidity increases gradually after $Y_{\mathrm{c}}$

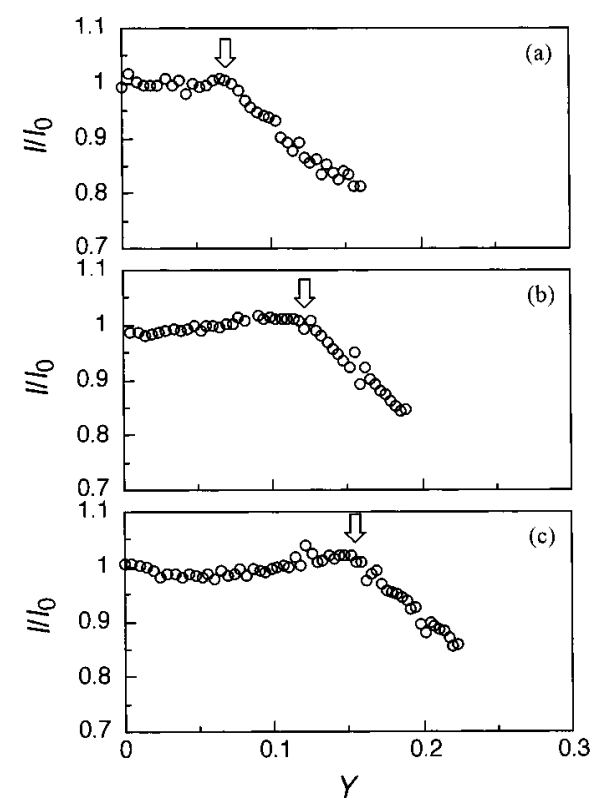

Figure 6. Type 1 fluorescence titration plots for solutions of $0.62 \mathrm{~g} / \mathrm{L}$ (4 mM monomer units) QPVP and $20 \mathrm{mM} \mathrm{C}_{12} \mathrm{E}_{8}$ in 0.1 (a), 0.2 (b), and $0.3 \mathrm{M} \mathrm{NaCl}$ (c), using a $40 \mathrm{mM}$ SDS solution of the same ionic strength as a titrant. Arrows denote $Y_{\mathrm{c}}$ values.

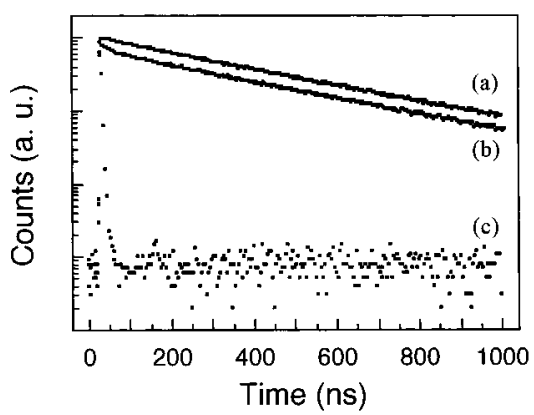

Figure 7. Fluorescence decay profiles for pyrene-carring $\mathrm{C}_{12} \mathrm{E}_{8} / \mathrm{SDS}$ mixed micelles of $Y=0$ (a) and 0.174 (b) in the presence of QPVP at $\mu=0.1$, accompanied by the lamp profile (c). $Y$ values were adjusted by addition of $40 \mathrm{mM}$ SDS in $0.1 \mathrm{M} \mathrm{NaCl}$ to a mixture of $0.62 \mathrm{~g} / \mathrm{L}$ QPVP, $20 \mathrm{mM} \mathrm{C}{ }_{12} \mathrm{E}_{8}$, and $4.6 \mu \mathrm{M}$ pyrene.

is reached. Figures 1 and 3 show that the turbidity increase is correlated with an increase in complex size. Thus, all of these data show a progressive increase in complex formation, which could be a fundamental characteristic of these systems or might arise in part from some system polydispersity that reduces the sharpness of the transition. One such polydispersity could be the compositional variability of the mixed micelles, but rather little information is currently available on this point. Recent work on the polyelectrolyte-binding of a single-surfactant micelle system ${ }^{15}$ suggests that a continuous increase in the binding constant beyond the critical micelle charge density is not limited to chemically mixed micelles but rather is characteristic of all related systems. For this reason, we describe the micelles to a first approximation only in terms of their average composition, i.e., the stoichiometric value of $Y$.

Time-Dependent Fluorescence Quenching for QPVP$\mathbf{C}_{12} \mathbf{E}_{\mathbf{8}} / \mathbf{S D S}$. Figure 7 shows an example of typical fluorescence decay profiles for pyrene-carrying $\mathrm{C}_{12} \mathrm{E}_{8} / \mathrm{SDS}$ mixed micelles of $Y=0$ and 0.174 in the presence of QPVP at $\mu=0.1$. Decays are completely single-exponential when $Y$ is small, but as $Y$ is increased, decays are best-fitted to a double-exponential function. However, a $Y$ value at which the transition occurs from 
TABLE 1: Lifetimes Determined by Fitting Fluorescence Decay Profiles

\begin{tabular}{clcccc}
\hline$\mu$ & $Y$ & $\tau_{0}{ }^{a}(\mathrm{~ns})$ & $\tau_{\mathrm{S}}(\mathrm{ns})$ & $\tau_{\mathrm{L}}(\mathrm{ns})$ & $\chi^{2}$ \\
\hline 0.1 & 0 & 379 & & 378 & 1.01 \\
0.1 & 0.09 & 384 & 41.7 & 377 & 1.23 \\
0.1 & 0.10 & 383 & 48.5 & 376 & 1.25 \\
0.1 & 0.12 & 384 & 37.6 & 375 & 1.18 \\
0.1 & 0.16 & 382 & 29.6 & 372 & 1.16 \\
0.1 & 0.17 & 382 & 39.1 & 371 & 1.10 \\
0.2 & 0 & 379 & & 381 & 1.12 \\
0.2 & 0.14 & 383 & 44.1 & 378 & 1.06 \\
0.2 & 0.16 & 385 & 34.1 & 376 & 1.24 \\
0.2 & 0.18 & 386 & 40.2 & 374 & 1.04 \\
0.2 & 0.20 & 384 & 43.8 & 372 & 1.07 \\
0.2 & 0.21 & 382 & 35.3 & 370 & 1.06 \\
0.3 & 0 & 383 & & 382 & 1.32 \\
0.3 & 0.17 & 384 & 78.2 & 381 & 1.14 \\
0.3 & 0.19 & 386 & 37.2 & 375 & 1.29 \\
0.3 & 0.21 & 387 & 35.3 & 374 & 1.12 \\
0.3 & 0.23 & 387 & 35.6 & 372 & 1.07 \\
0.3 & 0.24 & 387 & 35.0 & 370 & 1.24
\end{tabular}

${ }^{a}$ The fluorescence lifetime for pyrene-carrying mixed micelles in the absence of the quencher.

\section{SCHEME 1}

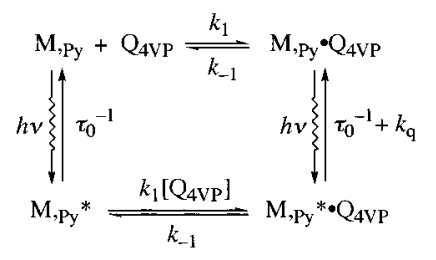

single- to double-exponential decay is not well-defined because, in the present system, fluorescence quenching efficiency is quite low. Above $Y_{\mathrm{c}}$, however, we can assume that these decay profiles obey a double-exponential function, as suggested from our previous study with PyPAMPS $-\mathrm{C}_{12} \mathrm{E}_{6} / \mathrm{CTAC}$ interactions. ${ }^{35}$ Thus, from the decay curves, the lifetimes of pyrene fluorescence were determined as shown in Table 1 , where $\tau_{\mathrm{S}}$ and $\tau_{\mathrm{L}}$ denote the shorter and longer lifetimes, respectively. At each $\mu, \tau_{\mathrm{L}}$ decreases as $Y$ is increased, although $\tau_{\mathrm{S}}$ seems to be independent of $Y$.

Kinetic Analysis for Fluorescence Quenching. To quantitatively interpret the fluorescence quenching data, we use a kinetic model that we proposed in our earlier work. ${ }^{35,36}$ This quenching model assumes an association equilibrium for the binding of pyrene-carrying $\mathrm{C}_{12} \mathrm{E}_{8} / \mathrm{SDS}$ mixed micelles to QPVP to form soluble complexes (Scheme 1). This assumtion is based on the observation that an association equilibrium is achieved in the region where soluble complexes are formed (Figure 4). Here, M,Py denotes the pyrene-carrying mixed micelles, $\mathrm{Q}_{4 \mathrm{VP}}$ denotes the pyridinium site in QPVP that acts as a quencher site, $\mathrm{M},{ }_{\mathrm{Py}} \cdot \mathrm{Q}_{4 \mathrm{VP}}$ denotes the complex of $\mathrm{M}$,Py with $\mathrm{Q}_{4 \mathrm{VP}}, k_{1}$ and $k_{-1}$ are the association and dissociation rate constants, respectively, $\tau_{0}$ is the fluorescence lifetime of pyrene in the absence of the quencher, and $k_{\mathrm{q}}$ is the first-order rate constant for fluorescence quenching within the complex. In this model, it is assumed that all pyrene probes are completely solubilized in micelles and hence no pyrene molecules exist in the bulk water phase. When the system is irradiated with UV light at equilibrium, pyrene probes in both free (uncomplexed) and complexed micelles are photoexcited, fluorescence quenching occurring only in the complexed micelles. However, free micelles having photoexcited pyrene may encounter QPVP within its fluorescence lifetime to form a complex, which may be followed by quenching within the complex. Here, we assume that the rate of deactivation of singlet-excited pyrene in the complex is much faster than the rate of dissociation of the complex, that is $\tau_{0}{ }^{-1}$ $+k_{\mathrm{q}} \gg k_{-1}$.

When the system is photoexcited by a light pulse at time $t=$ 0 , the total concentrations of the free and complexed micelles having photoexcited pyrene at time $t$ are given by a doubleexponential function with lifetimes $\tau_{1}$ and $\tau_{2}$ (eqs A4-A8 in Appendix). The reciprocals of these lifetimes are given by

$$
\left(1 / \tau_{1}\right)=\left(1 / \tau_{0}\right)+k_{\mathrm{q}}
$$

and

$$
\left(1 / \tau_{2}\right)=\left(1 / \tau_{0}\right)+k_{1}\left[\mathrm{Q}_{4 \mathrm{VP}}\right]
$$

On the other hand, the ratio of fluorescence quantum efficiencies in the presence and absence of QPVP is given by a function of $\tau_{0}, \tau_{1}, \tau_{2}$, and $k_{1}\left[\mathrm{Q}_{4 \mathrm{VP}}\right]$ (eq A12 in Appendix):

$$
\Phi / \Phi_{0}=\tau_{1} / \tau_{0}+\left(\tau_{2} / \tau_{0}\right)\left(1-\tau_{1} / \tau_{0}\right)\left\{1 /\left(1+K\left[\mathrm{Q}_{4 \mathrm{VP}}\right]\right)\right\}
$$

On the basis of steady-state fluorescence data $\left(\Phi / \Phi_{0}\right)$ and fluorescence decay data $\left(\tau_{0}, \tau_{1}\right.$, and $\left.\tau_{2}\right)$, one can calculate $K\left[\mathrm{Q}_{4 \mathrm{VP}}\right]$ using eq 4 . The quenching rate constant $k_{\mathrm{q}}$ and $k_{1}\left[\mathrm{Q}_{4 \mathrm{VP}}\right]$ can be calculated from fluorescence decay data using eqs 2 and 3 , respectively. From $K\left[\mathrm{Q}_{4 \mathrm{VP}}\right]$ and $k_{1}\left[\mathrm{Q}_{4 \mathrm{VP}}\right]$, the residence time $\left(1 / k_{-1}\right)$ of the micelle in the complex is calculated by

$$
1 / k_{-1}=K\left[\mathrm{Q}_{4 \mathrm{VP}}\right] / k_{1}\left[\mathrm{Q}_{4 \mathrm{VP}}\right]
$$

The concentration of the pyridinium site that can actually act as a quencher site ([ $\left.\mathrm{Q}_{4 \mathrm{VP}}\right]$ ) remains as an unknown parameter. Because the quencher sites are localized on the polymer chain, $\left[\mathrm{Q}_{4 \mathrm{VP}}\right]$ should be much lower than the molar concentration of the pyridinium residue of QPVP present in the system. In general, polymer-bound quenchers behave as much less efficient quenchers than do the corresponding free quencher moieties. In the quenching event in the $\mathrm{M}, \mathrm{Py} \cdot \mathrm{Q}_{4 \mathrm{VP}}$ complex, there exist excess quencher sites in the complex for photoexcited pyrene to be completely quenched. However, the excess quencher sites act as charged sites to interact with micellar charge, the pyridinium site playing a dual role as quencher and binding sites.

We applied this kinetic model to the steady-state and timedependent fluorescence data presented in Figure 6 and Table 1 , in which $\tau_{\mathrm{S}}$ and $\tau_{\mathrm{L}}$ correspond to $\tau_{1}$ and $\tau_{2}$, respectively. This set of data suggests that quenching efficiency of QPVP is quite low as compared with our earlier work. ${ }^{35,36} \mathrm{We}$ confirmed that all pyrene probes $(4.9 \pm 0.7 \mu \mathrm{M})$ are completely solubilized in the $\mathrm{C}_{12} \mathrm{E}_{8}$ micelle $(20 \mathrm{mM})$. Assuming that all pyrene probes are randomly distributed over the micelles according to a Poisson distribution, the average number of the probes solubilized in a micelle is calculated to be ca. 0.022 and the fraction of pyrenecarrying micelles to be ca. $0.022 \pm 0.003$. The rest of the micelles are pyrene-free. From the concentrations of the polymer $(0.62 \mathrm{~g} / \mathrm{L})$ and $\mathrm{C}_{12} \mathrm{E}_{8}$ molecules $(20 \mathrm{mM})$, along with the degree of polymerization for the polymer (ca. 730) and the aggregation number of the $\mathrm{C}_{12} \mathrm{E}_{8}$ micelle (ca. 90 ), ${ }^{43}$ the ratio of the molar concentrations of the micelle and the polymer molecule, [micelle]/[polymer], is roughly calculated to be ca. 40 , the micelle being in a large excess to the polymer molecule. This means that a considerable fraction of the polymers interact with pyrene-free micelles. This is another reason for the low quenching efficiency of QPVP. Moreover, the pyridinium sites may not be able to reach micelle cores where pyrene probes are solubilized because of an "excluded volume" effect between 


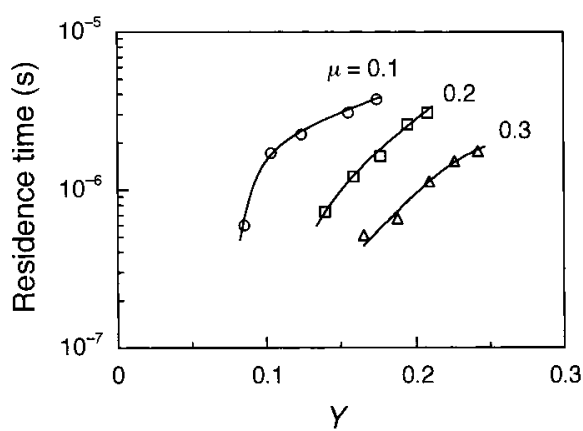

Figure 8. Plots of the residence time $\left(1 / k_{-1}\right)$ as a function of $Y$ for the $\mathrm{QPVP}-\mathrm{C}_{12} \mathrm{E}_{8} / \mathrm{SDS}$ system at $\mu=0.1,0.2$, and 0.3 .

the polymer chain and the ethylene oxide corona layer of the mixed micelles. Such a situation would lead to a decreased quenching efficiency.

Using eqs A4-A6 (Appendix) and 2-5, we calculated the values of $1 / k_{-1}$ at $\mu=0.1,0.2$, and 0.3 at varying $Y$. Results are plotted as a function of $Y$ in Figure 8. The residence time depends strongly on both the micelle charge density and the ionic strength. For example, at $\mu=0.1$, the residence time is ca. $0.6 \mu$ s at $Y=0.09$ (at a $Y$ slightly larger than $Y_{\mathrm{c}}$ ), but it increases to ca. $3.8 \mu$ s at $Y=0.17$, a 2-fold increase in the micelle charge density resulting in a more than 6 -fold increase in the residence time. More dramatically, the residence time increases from ca. 0.5 to ca. $3.8 \mu$ s upon decrease in the ionic strength from 0.3 to 0.1 , at $Y=0.17$. This increase in residence time by almost an order of magnitude upon a 2 -fold change in $\kappa$ is consistent with the results of recent Monte Carlo simulations. ${ }^{22,44}$ More dramatic effects may be expected with increasing colloid radius of curvature: Muthukumar ${ }^{45}$ has obtained the result that the residence time for a polyelectrolyte on a flat oppositely charged surface decreases by over an order of magnitude with only a $50 \%$ increase in $\kappa$.

Our earlier work revealed an interplay of hydrophobic and electrostatic interactions in complexation of PyPAMPS with quencher-carrying mixed micelles of $\mathrm{C}_{12} \mathrm{E}_{6} / \mathrm{CTAC} .{ }^{35}$ Despite the presence of hydrophobic effects, the residence times were strongly dependent on micelle charge and ionic strength, and the residence times were on the same order as those measured here, for comparable conditions. This suggests that the contribution of pyrene interaction with micelles to the residence times might be a secondary effect in the PyPAMPS system.

\section{Conclusions}

To eliminate hydrophobic contribution from fluorescence labels attached to polyelectrolytes in interactions between fluorescence-labeled polyelectrolytes and surfactant micelles, we employed QPVP in conjunction with mixed micelles of $\mathrm{C}_{12} \mathrm{E}_{8}$ and SDS in which pyrene probes are solubilized. Interactions of QPVP with pyrene-carrying $\mathrm{C}_{12} \mathrm{E}_{8} / \mathrm{SDS}$ mixed micelles were investigated by turbidimetric, quasielastic light scattering, and steady-state and time-dependent fluorescence techniques. The critical mole fractions of $\operatorname{SDS}\left(Y_{\mathrm{c}}\right)$, at which the formation of soluble polymer-micelle complexes starts to occur, were determined by turbidimetric and fluorescence techniques. The $Y_{\mathrm{c}}$ values determined by the two techniques are in good agreement. Dynamic interactions of QPVP with pyrene-carrying $\mathrm{C}_{12} \mathrm{E}_{8} / \mathrm{SDS}$ mixed micelles were investigated by steady-state and time-dependent fluorescence quenching techniques. The micelle charge density was varied systematically by varying the mole fraction of SDS $(Y)$ in the mixed micelle. Through the use of a kinetic model that we proposed previously as a first approximation, steady-state and time-dependent fluorescence quenching data were analyzed to estimate the residence time (the lifetime of a polymer-micelle dynamic complex). Results indicate that the residence time is a strong function of $Y$ and the ionic strength. In particular, a small decrease in the ionic strength causes a large increase in residence time.

Acknowledgment. P.D. acknowledges support from the National Science Foundation under Grant DMR0076068.

\section{Appendix}

According to Scheme 1, the concentration of the complex at equilibrium is given by

$$
\left[\mathrm{M}_{\mathrm{Py}} \cdot \mathrm{Q}_{4 \mathrm{VP}}\right]=K\left[\mathrm{M},{ }_{\mathrm{Py}}\right]\left[\mathrm{Q}_{4 \mathrm{VP}}\right]
$$

where $K$ is the association equilibrium constant (binding constant), i.e., $K=k_{1} / k_{-1}$.

Under transient conditions, the rate equations for $\left[\mathrm{M}, \mathrm{Py}^{*}\right]$ and $\left[\mathrm{M}, \mathrm{Py}^{*} \cdot \mathrm{Q}_{4 \mathrm{VP}}\right]$ with excitation at time $t=0$ by a light pulse of negligible duration are given by

$$
\begin{gathered}
\mathrm{d}\left[\mathrm{M},{ }_{\mathrm{Py}} *\right]_{t} / \mathrm{d} t=-\left(\tau_{0}{ }^{-1}+k_{1}\left[\mathrm{Q}_{4 \mathrm{VP}}\right]\right)\left[\mathrm{M},{ }_{\mathrm{Py}}{ }^{*}\right]_{t} \\
\mathrm{~d}\left[\mathrm{M},{ }_{\mathrm{Py}} * \cdot \mathrm{Q}_{4 \mathrm{VP}}\right]_{t} / \mathrm{d} t=-\left(\tau_{0}{ }^{-1}+k_{\mathrm{q}}\right)\left[\mathrm{M},{ }_{\mathrm{Py}} * \cdot \mathrm{Q}_{4 \mathrm{VP}}\right]_{t}+ \\
k_{1}\left[\mathrm{M},,_{\mathrm{Py}}{ }_{t}\right]_{t}\left[\mathrm{Q}_{4 \mathrm{VP}}\right]
\end{gathered}
$$

Solving eqs A2 and A3 with the initial conditions of $\left[\mathrm{M}, \mathrm{Py}^{*}\right]_{t}$ $=\left[\mathrm{M}, \mathrm{Py}{ }^{*}\right]_{t=0}$ at $t=0$ and $\left[\mathrm{M}, \mathrm{Py} * \cdot \mathrm{Q}_{4 \mathrm{VP}}\right]_{t}=\left[\mathrm{M}, \mathrm{Py} * \cdot \mathrm{Q}_{4 \mathrm{VP}}\right]_{t=0}$ at $t=0$, the total concentrations of $\left[\mathrm{M}, \mathrm{Py}^{*}\right]$ and $\left[\mathrm{M}, \mathrm{Py} * \cdot \mathrm{Q}_{4 \mathrm{VP}}\right]$ at time $t$ are given by

$$
\left[\mathrm{M},{ }_{\mathrm{Py}} *\right]_{t}+\left[\mathrm{M},{ }_{\mathrm{Py}} * \cdot \mathrm{Q}_{4 \mathrm{VP}}\right]_{t}=A \exp \left(-t / \tau_{1}\right)+B \exp \left(-t / \tau_{2}\right)
$$

where

$$
\begin{gathered}
A=\left[\mathrm{M},_{\mathrm{Py}} * \cdot \mathrm{Q}_{4 \mathrm{VP}}\right]_{t=0}\left\{1-k_{-1} /\left(k_{\mathrm{q}}-k_{1}\left[\mathrm{Q}_{4 \mathrm{VP}}\right]\right)\right\} \\
B=\left[\mathrm{M}_{\mathrm{Py}}{ }^{*}\right]_{t=0}\left\{k_{\mathrm{q}} /\left(k_{\mathrm{q}}-k_{1}\left[\mathrm{Q}_{4 \mathrm{VP}}\right]\right)\right\} \\
\left(1 / \tau_{1}\right)=\left(1 / \tau_{0}\right)+k_{\mathrm{q}}
\end{gathered}
$$

and

$$
\left(1 / \tau_{2}\right)=\left(1 / \tau_{0}\right)+k_{1}\left[\mathrm{Q}_{4 \mathrm{VP}}\right]
$$
by

Under steady-state conditions, the rate equations are given

$$
\begin{aligned}
\mathrm{d}\left[\mathrm{M},{ }_{\mathrm{Py}}{ }^{*}\right]_{t} / \mathrm{d} t=\left\{\left[\mathrm{M},{ }_{\mathrm{Py}}\right] /\left(\left[\mathrm{M},{ }_{\mathrm{Py}}\right]+\left[\mathrm{M},{ }_{\mathrm{Py}} \cdot \mathrm{Q}_{4 \mathrm{VP}}\right]\right)\right\} I_{\mathrm{a}}- \\
\left(\tau_{0}{ }^{-1}+k_{1}\left[\mathrm{Q}_{4 \mathrm{VP}}\right]\right)\left[\mathrm{M},{ }_{\mathrm{Py}}{ }^{*}\right]_{t} \\
\mathrm{~d}\left[\mathrm{M},,_{\mathrm{Py}} * \cdot \mathrm{Q}_{4 \mathrm{VP}}\right]_{t} / \mathrm{d} t=\left\{\left[\mathrm{M},{ }_{\mathrm{Py}} \cdot \mathrm{Q}_{4 \mathrm{VP}}\right] /\left(\left[\mathrm{M},{ }_{\mathrm{Py}}\right]+\right.\right. \\
{\left.\left.\left[\mathrm{M},,_{\mathrm{Py}} \cdot \mathrm{Q}_{4 \mathrm{VP}}\right]\right)\right\} I_{\mathrm{a}}+k_{1}\left[\mathrm{Q}_{4 \mathrm{VP}}\right]\left[\mathrm{M},{ }_{\mathrm{Py}}{ }^{*}\right]_{t}-} \\
\left(\tau_{0}{ }^{-1}+k_{\mathrm{q}}\right)\left[\mathrm{M},{ }_{\mathrm{Py}} \cdot \mathrm{Q}_{4 \mathrm{VP}}\right]_{t}(
\end{aligned}
$$

where $I_{\mathrm{a}}$ is the rate of light absorption. When $\mathrm{d}\left[\mathrm{M}, \mathrm{Py}^{*}\right]_{t} / \mathrm{d} t=0$ and $\mathrm{d}\left[\mathrm{M}, \mathrm{Py}^{*} \cdot \mathrm{Q}_{4 \mathrm{VP}}\right]_{t} / \mathrm{d} t=0$ are used, the total steady-state concentrations of $\left[\mathrm{M}, \mathrm{Py}^{*}\right]$ and $\left[\mathrm{M}, \mathrm{Py}^{*} \cdot \mathrm{Q}_{4 \mathrm{VP}}\right]$ are given by 


$$
\begin{array}{r}
{\left[\mathrm{M},{ }_{\mathrm{Py}}{ }^{*}\right]_{S}+\left[\mathrm{M},{ }_{\mathrm{Py}}{ }^{*} \cdot \mathrm{Q}_{4 \mathrm{VP}}\right]_{S}=\left\{\left[\mathrm{M},{ }_{\mathrm{Py}}\right] /\left(\left[\mathrm{M},{ }_{\mathrm{Py}}\right]+\right.\right.} \\
\left.\left.\left[\mathrm{M}, \mathrm{Py}_{\mathrm{Py}} \cdot \mathrm{Q}_{4 \mathrm{VP}}\right]\right)\right\} I_{\mathrm{a}}\left\{\left(1+K\left[\mathrm{Q}_{4 \mathrm{VP}}\right]\right)\left(\tau_{0}^{-1}+k_{1}\left[\mathrm{Q}_{4 \mathrm{VP}}\right]\right)+\right. \\
\left.k_{\mathrm{q}}\right\} /\left\{\left(\tau_{0}^{-1}+k_{1}\left[\mathrm{Q}_{4 \mathrm{VP}}\right]\right)\left(\tau_{0}^{-1}+k_{\mathrm{q}}\right)\right\}
\end{array}
$$

The ratio of fluorescence quantum efficiencies in the presence and absence of the quencher, $\mathrm{Q}_{4 \mathrm{VP}}$, is given by

$$
\Phi / \Phi_{0}=\tau_{1} / \tau_{0}+\left(\tau_{2} / \tau_{0}\right)\left(1-\tau_{1} / \tau_{0}\right)\left\{1 /\left(1+K\left[\mathrm{Q}_{4 \mathrm{VP}}\right]\right)\right\}
$$

\section{Note Added after ASAP Posting}

This article was released ASAP on 10/13/2001 with minor errors in eq 1 and the text following eq 1 . The correct version was posted on 10/19/2001.

\section{References and Notes}

(1) For reviews, see: (a) Tsuchida, E.; Abe, K. Adv. Polym. Sci. 1982, 45, 1. (b) Smid, J.; Fish, D. In Encyclopedia of Polymer Science and Engineering; Kroshwitz, J. I., Ed.; Wiley-Interscience: New York, 1988; Vol. 11, p 720.

(2) Xia, J.; Dubin, P. L.; Kim, Y. J. Phys. Chem. 1992, 96, 6805.

(3) Li, Y.; Dubin, P. L.; Dautzenberg, H.; Lück, U.; Hartmann, J.; Tuzar, Z. Macromolecules 1995, 28, 6795.

(4) Li, Y.; Xia, J.; Dubin, P. L. Macromolecules 1994, 27, 7049.

(5) Li, Y.; Dubin, P. L.; Havel, H. A.; Edwards, S. L.; Dautzenberg, H. Langmuir 1995, 11, 2486.

(6) Xia, J.; Zhang, H.; Rigsbee, D. R.; Dubin, P. L.; Shaikh, T. Macromolecules 1993, 26, 2759.

(7) Park, J. M.; Muhoberac, B. B.; Dubin, P. L.; Xia, J. Macromolecules 1992, 25, 290

(8) Tsuboi, A.; Izumi, T.; Hirata, M.; Xia, J.; Dubin, P. L.; Kokufuta, E. Langmuir 1996, 12, 6295.

(9) Wen, Y.-p.; Dubin, P. L. Macromolecules 1997, 30, 7856. (10) Mattison, K. W.; Dubin, P. L.; Brittain, I. J. J. Phys. Chem. B 1998, 102,3830

(11) Sato, T.; Mattison, K. W.; Dubin, P. L.; Kamachi, M.; Morishima, Y. Langmuir 1998, 14, 5430 .

(12) Li, Y.; Dubin, P. L.; Spindler, R.; Tomalia, D. Macromolecules 1995, 28, 8426 .

(13) Shah, G.; Dubin, P. L.; Kaplan, J. I.; Newkome, G. R.; Moorefield, C. N.; Baker, G. R. J. Colloid Interface Sci. 1996, 183, 397.

(14) Zhang, H.; Dubin, P. L.; Ray, J.; Manning, G. S.; Moorefield, C. N.; Newkome, G. R. J. Phys. Chem. B 1999, 103, 2347.

(15) Feng, X.; Dubin, P. L.; Kirton, G. Macromolecules 2001, 34, 6373.

(16) Zhang, H.; Ohbu, K.; Dubin, P. L. Langmuir 2000, 16, 9082

(17) Wiegel, F. W. J. Phys. A: Math. Gen. 1977, 10, 299.
(18) Evers, O. A.; Fleer, G. J.; Scheutjens, J. M. H. M.; Lyklema, J. J. Colloid Interface Sci. 1986, 111, 446.

(19) Muthukumar, M. J. Chem. Phys. 1987, 86, 7230.

(20) Odijk, T. Langmuir 1991, 7, 1991.

(21) von Goeler, F.; Muthukumar, M. J. Chem. Phys. 1994, 100, 7796.

(22) (a) Muthukumar, M. J. Chem. Phys. 1995, 103, 4723. (b) Chodanowski, P.; Stoll, S. Macromolecules 2001, 34, 2320.

(23) Dubin, P. L.; Rigsbee, D. R.; McQuigg, D. W. J. Colloid Interface Sci. 1985, 105, 509 .

(24) McQuigg, D. W.; Kaplan, J. I.; Dubin, P. L. J. Phys. Chem. 1992 96, 1973.

(25) Dubin, P. L.; Oteri, R. J. Colloid Interface Sci. 1983, 95, 453.

(26) Dubin, P. L.; Rigsbee, D. R.; Gan, L. M.; Fallon, M. A. Macromolecules 1988, 21, 2555.

(27) Jones, M. N. J Colloid Interface Sci. 1967, 23, 36.

(28) Rigsbee, D. R.; Dubin, P. L. Langmuir 1996, 12, 1928

(29) Sudbeck, E. A.; Dubin, P. L.; Curran, M. E.; Skelton, J. J. Colloid Interface Sci. 1991, 142, 512.

(30) Tokiwa, F.; Tujii, K. Bull. Chem. Soc. Jpn. 1973, 46, 2684.

(31) Fishman, M. L.; Eirich, F. R. J. Phys. Chem. 1971, 75, 3135.

(32) Shirahama, K. Colloid Polym. Sci. 1974, 252, 978.

(33) Yoshida, K.; Morishima, Y.; Dubin, P. L.; Mizusaki, M. Macromolecules 1997, 30, 6208.

(34) Mizusaki, M.; Morishima, Y.; Yoshida, K.; Dubin, P. L. Langmuir 1997, 13, 6941 .

(35) Morishima, Y.; Mizusaki, M.; Yoshida, K.; Dubin, P. L. Colloids Surf., A 1999, 147, 149.

(36) Mizusaki, M.; Morishima, Y.; Dubin, P. L. J. Phys. Chem. B 1998, $102,1908$.

(37) Chu, D.-Y.; Thomas, J. K. Macromolecules 1984, 17, 2142.

(38) Poly(4-vinylpyridine) was quaternized by methyl iodide in sulfolane under an argon atmosphere to form $\operatorname{poly}(N$-methyl-4-vinylpyridinium iodide). Because iodide may quench pyrene fluorescence, the iodide was exchanged to chloride as follows: the iodide was exchanged to perchlorate by using silver perchlorate, and then the perchlorate was exchanged to chloride by dialysis against dilute hydrochloric acid $(\mathrm{pH}=2.0)$ and by using an anion-exchange resin.

(39) Dubin, P. L.; Davis, D. Colloids Surf. 1985, 13, 113.

(40) Dubin, P. L.; The, S. S.; McQuigg, D. W.; Chew, C. H.; Gan, L. M. Langmuir 1989, 5, 89.

(41) Dubin, P. L.; Chew, C. H.; Gan, L. M. J. Colloid Interface Sci. 1989, $128,566$.

(42) Yoshida, K.; Sokhakian, S.; Dubin, P. L. J. Colloid Interface Sci. 1998, 205, 257.

(43) van Os, N. M.; Haak, J. R.; Rupert, L. A. M. Physico-Chemical Properties of Selected Anionic, Cationic and Nonionic Surfactants; Elsevier: Amsterdam, 1993.

(44) Some simulations of polyelectrolyte-micelle complexes have identified structural features without observation of any phase-transitionlike behavior: Wallin, T.; Linse, P. Langmuir, 1996, 12, 305; J. Phys. Chem. 1996, 100, 17873.

(45) M. Muthukumar, University of Massachusetts, Amherst, MA Private communication, 1997. 